\title{
MARKET POWER SCREENS WILLINGNESS-TO-PAY*
}

\author{
E. Glen Weyl $\quad$ Jean Tirole ${ }^{\ddagger}$
}

June 8, 2012

\begin{abstract}
What is the best way to reward innovation? While prizes avoid deadweight loss, intellectual property selects high social surplus projects. Optimal innovation policy thus trades off the ex-ante screening benefit and the ex-post distortion. It solves a multidimensional screening problem in the private information held by the innovator: research cost, quality and market size of the innovation. The appropriate degree of market power is never full monopoly pricing and is determined by measurable market characteristics, the inequality and elasticity of innovation supply, making the analysis open to empirical calibration. The framework has applications beyond IP policy to the optimal pricing of platforms or the optimal procurement of public infrastructure.
\end{abstract}

JEL Codes: D40, D61, D82, L10, L40

Keywords: intellectual property, market power, screening, stretch parametrization.

${ }^{*}$ Weyl acknowledges the support of the Milton Fund and Nokia. Tirole acknowledges the support of Microsoft and Nokia to the Institute for Industrial Economics (IDEI). The research leading to these results has received funding from the European Research Council under the European Community's Seventh Framework Programme (FP7/2007-2013) Grant Agreement no. 249429. We are grateful to many people for the helpful comments they provided on this paper, but we especially thank Roland Bénabou, Joshua Gans, Elhanan Helpman, Scott Duke Kominers, José Scheinkman, Josh Schwartzstein, Yossi Spiegel, Scott Stern, Michael Whinston, Pai-Ling Yin, and four anonymous referees. We also appreciate the excellent research assistance of Stephanie Lo, Vladimir Mukharlyamov, David Smalling, William Weingarten and especially André Veiga and Rui Wang. All errors are our own.

${ }^{\dagger}$ Department of Economics, University of Chicago, 1126 E. 59th Street, Chicago, IL 60637, USA, and Toulouse School of Economics, 21, allée de Brienne, Toulouse 31000, France. weyl@uchicago.edu. The order of authors has been chosen to conform to the scientific convention of priority of contribution rather than the social convention of alphabetic precedence.

‡TSE, 21, allée de Brienne, Toulouse 31000, France: jean.tirole@tse-fr.eu. 
Can innovation be rewarded without the distortions created by intellectual property (IP)?1 At least since Marshall (1890), textbook economics has advocated replacing IP with a system of prizes (in which the innovator receives a lump sum and the innovation is put in the public domain) and, more broadly, replacing monopoly by marginal cost pricing. Yet an even older tradition (Smith, 1762) has argued that without market-driven rewards, i.e., without the exercise of market power, it is difficult to determine which projects merit the necessary development costs. Refining the Marshallian argument, Kremer (2000a , b b ) points out that basing prizes on consumption at the efficient price avoids both the ex-post pricing distortion and the waste of resources on useless innovations 2 Yet the use of market size in these "quantity-dependent prizes" ignores a second dimension of heterogeneity among innovations, also necessary to determine their social value: consumers' willingness to pay for them.

This paper analyzes the tradeoff between the screening benefit of market power and the traditional pricing distortion in a framework in which entrepreneurs have multidimensional private information. Our model, developed in Section I features an entrepreneur who is privately informed about the size $\sigma$ of the market for her innovation, its quality $m$ (indexing consumers' willingness to pay for it) and the fixed cost $c$ of bringing it about.

Figure \illustrates the basic idea. Consider two equally costly innovations, with demand curves $D_{2}$ ("me-too") and $D_{1}$ ("major"). Because willingnesses to pay are small, "innovation $D_{2}$ " creates little social value despite its large market size and does not justify the fixed cost $c$ of bringing it about. An example might be Netscape Navigator during the 1990's which, while widely adopted, sold at a low price because it offered little added value over its rivals. In contrast, America OnLine (AOL), depicted by innovation $D_{1}$ in Figure 1 had a smaller customer base $\left(\sigma_{1}<\sigma_{2}\right)$ but brought substantial value to its consumers (and charged a correspondingly

\footnotetext{
${ }^{1}$ IP may take many forms: trade secrets, copyrights, patents, etc. We follow much recent literature in seeing the broad institution of market power as a reward for innovation (regardless of the exact form it takes) as separate from the specific institution of patents; our focus is on whether market power is appropriate, however implemented.

${ }^{2}$ Interest in centralized systems for stimulating innovation, including Kremer's "advance market commitments" (Barder et al., 2005), has risen in recent years with the resurgence of industrial policy (Economist, 2010).

Despite the extensive theoretical and policy interest in, and numerous successful historical examples (Kremer, 1998) of the prize system, many consider it simply impractical. Yet it is hard to see what, other than informational asymmetries, could be the source of such "impracticality". Perhaps there is simply a fixed cost of government involvement; but then this could not explain the patent system, which clearly involves the government. Furthermore, market power is used in many situations, such as infrastructure procurement, where government involvement is inevitably highly intrusive; see also our application to platform markets in Subsection IV.B.
} 
high price).

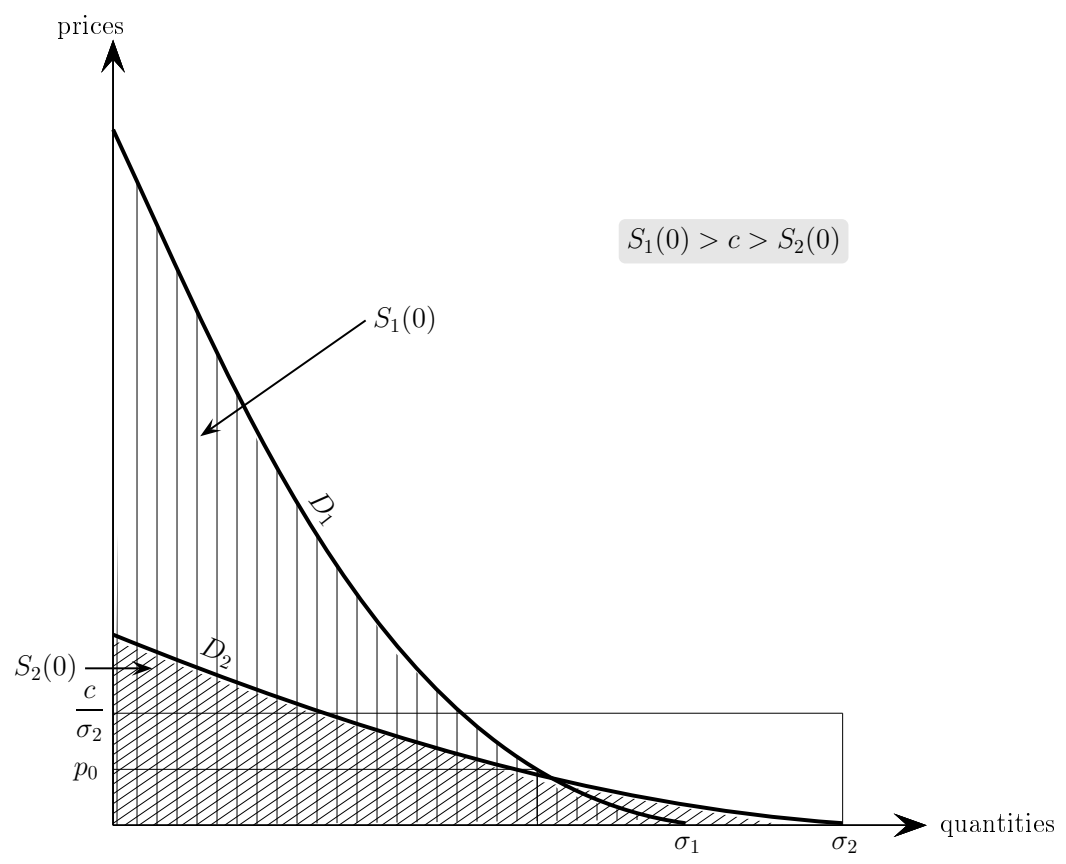

Figure I: Distinguishing valuable from low-surplus projects. $S_{i}(0)$ denotes the gross consumer surplus for demand $D_{i}$ at the ex-post efficient price 0.

A social planner who does not directly observe consumer surplus can separate innovation $D_{2}$ from innovation $D_{1}$ provided that the demand for a higher-quality product is less sensitive to price. This requires charging a price for $D_{1}$ of at least $p_{0}$, the minimum price at which $D_{1}$ actually exceeds $D_{2}$. At lower prices, $D_{2}$ looks superior to $D_{1}$, being more widely adopted. Fundamentally, a quantity-dependent prize system - a payment to the entrepreneur depending only on demand at marginal cost (say 0 , or more generally below $p_{0}$ ) - is unable to screen out $D_{2}$ and screen in $D_{1}$. By contrast, IP can screen in the good innovation and out the bad one if monopoly profits $\left(\pi_{i}=\max p D_{i}(p)\right)$ obey $\pi_{1}>c>\pi_{2}$.

Section \introduces a stretch parameterization of general demand function $Q$,

$$
q=\sigma Q\left(\frac{p}{m}\right)
$$

where the quality parameter $m$ is normalized so as to denote the monopoly price. While, as we show, our analysis applies more broadly than the stretch parameterization, this parameterization is particularly handy due to its property of proportionality of average consumer surplus 
and profit when the price is equal to a fixed fraction of the monopoly price.

A social planner only has a prior over $(\sigma, m, c)$ and seeks to maximize total social welfare. Because we observe institutions (such as output subsidies, price controls and competition spurred by weak IP) that help limit prices without achieving full ex-post efficiency, we grant our social planner access to a range of policies between quantity-dependent prizes and IP. The entrepreneur's net reward $t(q, p)$ depends on her choice of output and price. Normalizing marginal cost at 0 , this class includes standard approaches to rewarding innovation: prizes $\left(t(q, p)=t_{0}\right)$, Kremer quantity-dependent prizes $(t=t(q)$, an increasing function of $q$, leading to a transfer based on quantity sold at marginal cost), and patents $(t(q, p)=q p$, leading to monopoly pricing and output).

While our online Appendix shows that similar insights can be obtained for general reward functions $t(q, p)$, the paper focuses on a simple class of policies with Cobb-Douglas isoreward curves $t\left(q^{1-\alpha} p^{\alpha}\right)$. This class indeed moves continuously from a Kremer quantity-dependent prize system $\left(t=t\left(q^{1} p^{0}\right)\right)$ to a generalized patent system $\left(t=t\left(q^{1 / 2} p^{1 / 2}\right)\right)$ as the parameter $\alpha$ that indexes the structure of the reward increases from 0 to $1 / 2$; values above $1 / 2$ will turn out to be suboptimal.

Section [1] demonstrates that raising $\alpha$ at the margin selects in high quality and out high market size innovations. However, it also increases deadweight loss on all innovations brought to market, not just those on the margin between being created or not. Thus the relative benefit of screening also depends on the relative number of marginal and inframarginal innovations.

We show that an increase in market power achieves better sorting/screening - it selects better (higher social surplus) innovations - so long as $\alpha<1 / 2$. This result formalizes Smith (1762)'s hypothesis that the monopoly profit is "a pecuniary reward...precisely proportional to the merit of the invention." Pure IP $(\alpha=1 / 2)$ yields perfect sorting and is never optimal as the marginal sorting benefits dwindle while the marginal deadweight losses do not; pure quantity-dependent prizes $(\alpha=0)$ are, under a weak condition, also never optimal as the marginal deadweight loss is 0 at the ex-post-efficient price.

Section III then quantifies this trade-off between ex-post distortion and screening. Nearmonopoly prizes are optimal when the product of the following two quantities is large: 
- elasticity of innovation supply. Inducing higher prices reduces the social value of all innovations, not just marginal ones. And so the screening benefit-deadweight loss cost ratio is higher, the higher the ratio of marginal to inframarginal innovations. Screening through high prices is therefore attractive when innovation supply is highly elastic.

- inequality of innovation supply. Intuitively, screening is more desirable, the more dispersed the quality of innovations for given observables $(p, q$, and other relevant information). We provide a precise definition of this dispersion or inequality of innovation supply. For close-to-monopoly price, it is equal to the variance of $\log (m / \sigma) 3$ Furthermore, the ratio $m / \sigma$ is equal to $p / q$ in equilibrium, and so the inequality of innovation supply can be empirically estimated.

The sorting role of market power arises not only in the provision of incentives for innovation. Other leading examples include bundling vs. unconstrained pricing in online platforms and media markets, incentives for product development within firms and cost recovery rules in public infrastructure procurement. Accordingly, Section IV discusses applications and implementation of our analysis in these contexts. Section $\mathrm{V}$ concludes the paper with a discussion of directions for future research. More technical aspects of the analysis are discussed in two appendices following the main text. Longer and less instructive proofs, as well as an extension of the results beyond the Cobb-Douglas isoreward class and a treatment of our problem based on the revelation principle, appear in an online appendix at http://www.glenweyl.com.

Related literature. Previous literature on the design of institutions for rewarding innovation has either ruled out prizes or considered environments with a single dimension of heterogeneity in which quantity-dependent prizes achieve optimal sorting. The first strand of literature assumes either explicitly that no transfers can be made from the planner to the entrepreneur (Gilbert and Shapiro, 1990; Klemperer, 1990; Green and Scotchmer, 1995; O'Donoghue et al., 1998; Scotchmer, 1999; Hopenhavn and Mitchell, 2001), ruling out prizes and R\&D or output

subsidies, or that the asymmetry of information is so severe that prizes can only drain the plan-

\footnotetext{
${ }^{3}$ In fact, the variance of the logarithm is one of the most commonly used measures of inequality (Creedy, 1977; Foster and Ok, 1999). It is particularly intuitive in our setting because its well-known drawback of being hard to ground in utility theory (Dasgupta et al., 1973) is irrelevant, while its primary benefit of emphasizing the degree of extremely low and high outcomes $(\underline{\operatorname{Sen}}, 1973)$ is central to capturing the idea that a few breakthrough innovations may weigh a lot in the global surplus brought about by R\&D. We thank Nicolas Pistolesi for these references.
} 
ner's resources (Cornelli and Schankerman, 1999; Gallini and Scotchmer, 2002; Hopenhavn et al., 2006; Chari et al., 2009). Thus this literature has focused on the allocation of market power across time or product space, rather than on its desirability per se. The second strand of literature allows for prizes, but only a single dimension of heterogeneity, in which case quantitydependent prizes screen perfectly. This work thus either compares quantity-independent prizes and IP (Shavell and van Ypersele, 2001; Wright, 1983), or concludes that quantity-dependent prizes achieve the first-best (Kremer, 2000a , b). By allowing multidimensional heterogeneity, our framework creates a smooth trade-off between screening and ex-post pricing distortion that determines which of a range of institutions running between IP and quantity-dependent prizes is optimal.

\section{SET-UP}

\section{(a) Innovations.}

Although our analysis is more general (see section [II.D), its essence is captured by a simple framework, in which potential innovations, each independent in production and consumption, are characterized by three numbers:

- $\sigma$, the size of the market, i.e., the number of potentially interested users, as measured by demand at $p=0$;

- $m$, the quality of the innovation;

- and $c$, the ex-ante cost of creating the innovation.

The innovation's ex-post marginal cost is 0 . Demand is given by

$$
q=\sigma Q\left(\frac{p}{m}\right)
$$

and has elasticity

$$
\epsilon(a) \equiv-a Q^{\prime}(a) / Q(a), \text { where } a \equiv \frac{p}{m} .
$$

Normalization. We normalize $Q(0)=1$ (thereby requiring finite demand at $p=0$ ), so ex-postefficient demand is $\sigma$, and $\epsilon(1)=1$ so that the monopoly optimal price is $m$. 
Regularity assumptions. $Q$ is assumed smooth, strictly decreasing wherever it is strictly positive, to have strictly declining marginal revenue and bounded $\frac{\epsilon^{\prime \prime}}{\epsilon}$, and to have vanishing marginal distortion at the ex-post-efficient price $\left(\lim _{a \rightarrow 0} a Q^{\prime}(a)=0\right)$ implying $\varepsilon(0)=0$. For marginal revenue to be decreasing, $\epsilon^{\prime}>0$ whenever $\epsilon \leq 1$ and $\varepsilon>1$ for $a>1$. 4

A simple example is linear demand $q=\sigma(2 m-p) / 2 m ; \sigma$ corresponds to the quantityintercept of linear demand and $m$ to half of the price-intercept. More generally, $\sigma$ represents a horizontal stretching of inverse demand while $m$ is a vertical stretching. We thus refer to this as the stretch parametrization 5 A key feature of the stretch parametrization is the proportionality between average social surplus and monopoly profit when the same fraction $a$ of the monopoly price is charged for all innovations (the robustness of our results to relaxing this assumption is discussed in Subsection (III.D). Indeed, the gross social surplus created by an innovation is

$$
\underbrace{p \sigma Q\left(\frac{p}{m}\right)}_{\text {profit }}+\underbrace{\sigma \int_{p}^{\infty} Q\left(\frac{\tilde{p}}{m}\right) d \widetilde{p}}_{\text {net consumer surplus }}=\sigma m\left(a Q(a)+\int_{a}^{\infty} Q(\tilde{a}) d \widetilde{a}\right) \equiv \sigma m S(a)
$$

Thus if $a$ is constant across types (as will later be shown for Cobb-Douglas reward functions), so is the ratio of social surplus to profit $S(a) / a Q(a)$.

\section{(b) Information, payoffs and timing.}

Each innovation is owned by an entrepreneur, who has private information about $\theta=$ $(\sigma, m, c)$. The social planner only knows that the entrepreneur's "type" $\theta$ is distributed according to some smooth pdf $f$ with full support $\mathbb{R}_{++}^{3}$ and all moments finite. The social planner, who will ex post observe price and output, designs a net reward $t(q, p)$ for the innovator 6 We

$$
\begin{aligned}
& { }^{4} M R=p-\frac{p}{\epsilon} \propto a-\frac{a}{\epsilon(a)}, \text { so } \\
& Q^{\prime}(M R)^{\prime}>0 \Longleftrightarrow\left[a\left(1-\frac{1}{\epsilon}\right)\right]^{\prime}<0 \Longleftrightarrow \epsilon^{\prime}>-\frac{\epsilon}{a}\left(1-\frac{1}{\epsilon}\right) \Longleftrightarrow \epsilon^{\prime}>-\frac{\epsilon^{2}}{a}\left(1-\frac{1}{\epsilon}\right) .
\end{aligned}
$$

${ }^{5}$ An increase in $\sigma$ corresponds to an increase in the traditional market size parameter in standard industrial organization (Bresnahan and Reiss, 1991) and international trade (Krugman, 1980). A shift in $m$, on the other hand, corresponds to a proportional increase in all consumers' willingness-to-pay, often represented (Berry et al., 1995) by a proportional increase in their income.

${ }^{6}$ We adopt the convention that the social planner receives the profit $p q$. Equivalently, and as is the case in practice, the entrepreneur keeps the profit, and the subsidy (or tax) is $t(q, p)-p q$. 
require that $t$ be non-decreasing in its arguments 7

The entrepreneur's utility is $t-c$ if she innovates and 0 otherwise. The social planner's utility is equal to net social surplus, $\sigma m S(a)-c$.

The timing is described in Figure

\begin{tabular}{lll}
\hline The social planner & The entrepreneur learns & The entrepreneur (if she \\
designs net reward & her type $\theta=(\sigma, m, c)$ & has innovated) chooses \\
mechanism $t(q, p)$ & $\begin{array}{l}\text { and decides whether to } \\
\text { innovate }\end{array}$ & $\begin{array}{l}\text { her price, and receives } \\
\text { reward } t(q, p)\end{array}$
\end{tabular}

Figure II: Timing

If she innovates, the entrepreneur chooses $p$ so as to maximize her reward $t\left(\sigma Q\left(\frac{p}{m}\right), p\right)$. Equivalently, she chooses her optimal fraction of the monopoly price $a^{\star}(\sigma, m ; t(\cdot, \cdot))$. She creates the innovation if $c$ is smaller than the resulting reward. The social planner chooses the net reward schedule $t(q, p)$ so as to maximize the total social welfare created by innovation:

$$
\int_{\theta: c \leq t\left(\sigma Q\left(a^{\star}(\sigma, m ; t(\cdot, \cdot))\right), m a^{\star}(\sigma, m ; t(\cdot, \cdot))\right)}\left[\sigma m S\left(a^{\star}(\sigma, m ; t(\cdot, \cdot))\right)-c\right] f(\theta) d \theta
$$

Before deriving the optimal mechanism in Section 【I, we explain in Section $\amalg$ a convenient approach for solving this multidimensional problem.

\section{The IsOREWARD APPROACH}

It is useful to decompose policies into their structure and level of rewards. Each policy $t(q, p)$ is characterized by its isoreward curves, and the actual level of rewards it assigns to each of these curves. As pictured in Figure III, to maximize her reward, the entrepreneur chooses the point on her demand curve at which it is tangent to an isoreward curve. Thus, only the isoreward curves, and not the levels of reward assigned to them, affect pricing incentives. The levels of reward affects only the entrepreneur's decision to enter. We refer to this decomposition as the isoreward approach.

\footnotetext{
${ }^{7}$ This must be so if there is free disposal (the entrepreneur can throw away output/refuse to serve). Then $t$ must be non-decreasing in $q$. Imagine further that for some $q, p_{L}, p_{H}$ with $p_{L}<p_{H}, t\left(q, p_{L}\right)>t\left(q, p_{H}\right)$; then an entrepreneur with demand $q$ at price $p_{H}$ could charge $p_{L}$, throw away the increase in output, and make more money.
} 


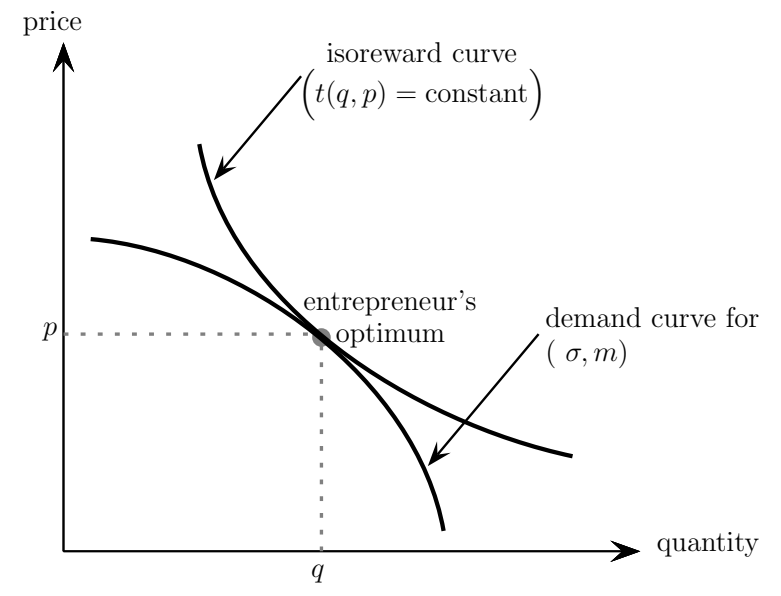

Figure III: The isoreward approach

The applicability of the isoreward approach requires only very mild conditions: see our online appendix. However, for expositional purposes, we find it useful to restrict attention to Cobb-Douglas isoreward policies, $t=t\left(q^{1-\alpha} p^{\alpha}\right)$. This parsimonious single-parameter class allows empirical calibration while providing a continuous map between the extremes of generalized IP $(\alpha=1 / 2)$ and quantity-dependent prizes $(\alpha=0)$.

Another useful property of the Cobb-Douglas isoreward class is that, given the assumed stretch parameterization, the innovator is incentivized to charge the same fraction $a$ of the monopoly price regardless of her type:

$$
\max _{\{q, p\}} t\left(q^{1-\alpha} p^{\alpha}\right) \text { s.t. } q=\sigma Q\left(\frac{p}{m}\right) \Longleftrightarrow \max _{\{a\}}\left\{Q(a)^{1-\alpha} a^{\alpha}\right\} .
$$

Cobb-Douglas isoreward curves are particularly simple from this perspective as they have a constant elasticity $\frac{\alpha}{1-\alpha}$. Thus the entrepreneur chooses a value of $a$ that equates the elasticity of her demand curve, $\epsilon(a)$, to $\frac{\alpha}{1-\alpha}$ : Regardless of type, the entrepreneur charges the same, unique $a(\alpha)$ solving

$$
\epsilon(a(\alpha))=\frac{\alpha}{1-\alpha}
$$

leading to a price below the monopoly level for $\alpha \in[0,1 / 2)$, and above the monopoly level for $\alpha>1 / 2$ (we will show that $\alpha \geq 1 / 2$ is never optimal, though). Furthermore for $\alpha<1 / 2$, $\epsilon^{\prime}>0$ and so $a^{\prime}>0$ : Price and ex-post distortion rise in $\alpha$. We can thus refer to $\alpha$ as the degree of market power chosen by the planner.

Because the entrepreneur charges the same $a$ regardless of type, the price and quantity are 
proportional to $m$ and $\sigma$ respectively as $p=a(\alpha) m$ and $q=\sigma Q(a(\alpha))$. Thus an entrepreneur of type $(\sigma, m)$ receives reward

$$
\tau\left(\sigma^{1-\alpha} m^{\alpha} ; \alpha\right) \equiv t\left(Q(a(\alpha))^{1-\alpha} a(\alpha)^{\alpha} \sigma^{1-\alpha} m^{\alpha}\right) .8
$$

Additionally, the value of the innovation, $\sigma m S(a(\alpha))$ is proportional to $\sigma m$. Thus, Pigovian payment in accordance to product would require the reward to be based on $\sigma m$, or equivalently

on $\sigma^{\frac{1}{2}} m^{\frac{1}{2}}$, and thus is possible only with $\alpha=\frac{1}{2}$ (i.e. full monopoly pricing). The higher the value of $\alpha$ (below $\frac{1}{2}$ ), the closer we are to payment in accordance with product and thus to perfect sorting among innovations. But a higher $\alpha$ also leads to a higher ex-post distortion. This establishes the basic tradeoff between sorting and ex-post efficiency that is the key to optimal policy.

This also immediately implies a useful and intuitive result: It is never optimal to choose $\alpha>\frac{1}{2}$ and thus to induce prices above the monopoly optimum. This both worsens ex-post distortion and reduces the quality of sorting by over-rewarding quality relative to size.

\section{Optimal ReWARds AND PRICING}

The planner chooses market power and a transfer schedule so as to maximize expected gross consumer surplus minus R\&D cost, given the entrepreneur's response:

$$
\max _{\alpha}\{W(\alpha)\} \text { where } W(\alpha) \equiv \max _{\tau(\cdot ; \alpha)} \int_{\theta: c \leq \tau\left(\sigma^{1-\alpha} m^{\alpha} ; \alpha\right)}[\sigma m S(a(\alpha))-c] f(\theta) d \theta
$$

To solve this program, we employ a two-step strategy. First, we fix market power $\alpha$ (or equivalently a) and maximize social welfare over choices of reward schedule $\tau$. Then after establishing the applicability of the envelope theorem to our context and thus the differentiability of the value function $W(\alpha)$, we use the envelope condition to determine the marginal benefit and cost of raising $\alpha, W^{\prime}$.

\footnotetext{
${ }^{8}$ From here on we use $\tau$ to represent rewards in the type space and $t$ the same in price-quantity space.
} 


\section{III.A. Optimal transfers}

We first consider the optimal reward along each isoreward curve independently, a simple unidimensional problem. It is useful to re-parameterize the problem in terms of isoreward curves, indexed by $k \in[0, \infty)$ and by the quality-market size ratio $x$,

$$
k=\sigma^{1-\alpha} m^{\alpha} \quad \text { and } \quad x \equiv \frac{m}{\sigma},
$$

rather than in terms of $(\sigma, m)$. We will refer to the relevant (change-of-variables augmented) distribution function as $\tilde{f}(k, x, c)$ to distinguish it from $f(\sigma, m, c)$. The change of variables from $(\sigma, m)$ to $(k, x)$ transforms the distribution of values according to

$$
\tilde{f}(k, x, c ; \alpha) \equiv f\left(k x^{-\alpha}, k x^{1-\alpha}, c\right) k x^{-2 \alpha} .
$$

In this new notation, the social value created by an innovation can be rewritten as

$$
\sigma m S(a(\alpha))=k^{2} x^{1-2 \alpha} S(a(\alpha)) \text {. }
$$

For given isoreward curve $k$ and associated reward $\tau(k ; \alpha)$, all innovations along the isoreward curve with cost less than $\tau(k ; \alpha)$, i.e., those with $c \leq \tau\left(\sigma^{1-\alpha} m^{\alpha} ; \alpha\right)$, are created. If the "average marginal innovation" (i.e. the average innovation along isoreward curve $k$ with cost $c=\tau(k ; \alpha))$ creates social value greater (lower) than $\tau(k ; \alpha)$, the social planner has an incentive to raise (lower) $\tau(k ; \alpha)$. Thus for a given $\alpha$ and $k$, the optimum is at a point at which these are exactly equated:

$$
\tau^{\star}(k ; \alpha)=k^{2} S(a(\alpha)) E\left(x^{1-2 \alpha} \mid c=\tau^{*}(k ; \alpha), k\right),
$$

where the expectation is taken over $x$ under $\tilde{f}$. If condition (3) delivers a unique and nondecreasing-in- $k$ function $\tau^{*}$, then it characterizes the optimal transfer policy for each isoreward curve 9 The optimal transfer function in the $(q, p)$ space is then derived simply by inverting

\footnotetext{
${ }^{9}$ If $c$ is not too affiliated with $x$ under $\widetilde{f}$ given $k$, in a sense formalized in Proposition 1 in Appendix A then there is a unique point at which this condition is satisfied. Furthermore, if $k$ is "not too negatively affiliated under $\widetilde{f}$ with $x$ given $c$ " then $\tau^{\star}$ is monotone increasing and thus is the truly optimal transfer function. If either (but not both)
} 
the definition of $\tau: t^{\star}\left(Q(a(\alpha))^{1-\alpha} a(\alpha)^{\alpha} k\right)=\tau^{\star}(k ; \alpha)$.

\section{III.B. Optimal market power}

Because $\tau^{\star}(k ; \alpha)$ is chosen optimally, we can apply Milgrom and Segal (2002)'s envelope theorem for general choice sets and consider only the direct effect of an increase in $\alpha$ on social welfare, ignoring indirect effects through the optimal choice of $\tau^{\star}$.

Lemma 1. $W(\alpha)$ is differentiable for all $\alpha \in\left(0, \frac{1}{2}\right)$ and its derivative may be evaluated by the envelope theorem, holding $\tau^{\star}$ fixed. Formally:

$$
W^{\prime}(\hat{\alpha})=\left.\frac{\partial}{\partial \alpha}\left[\int_{\sigma} \int_{m} \int_{c=0}^{\tau^{\star}\left(\sigma^{1-\alpha} m^{\alpha} ; \hat{\alpha}\right)}[\sigma m S(a(\alpha))-c] f(\sigma, m, c) d c d m d \sigma\right]\right|_{\alpha=\hat{\alpha}}
$$

Proof. See Online Appendix, Section 3.

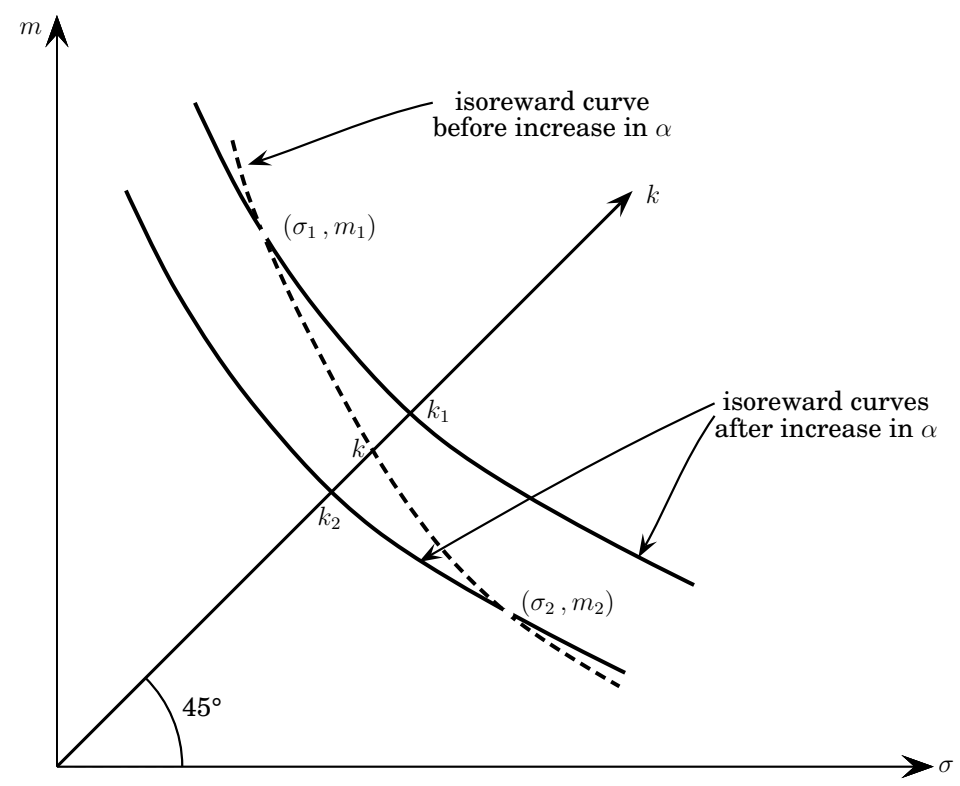

Figure IV: Increasing market power selects in high-quality innovations

\section{Heuristics.}

Figure IV first depicts an isoreward curve (the dashed curve) for a given $\alpha$. Types $\left(\sigma_{1}, m_{1}\right)$ and $\left(\sigma_{2}, m_{2}\right)$ receive the same reward. An increase in $\alpha$ makes the isoreward curves flatter.

of these conditions fails, standard ironing techniques can be used to determine optimal transfers as described in Appendix $\underline{\mathrm{A}}$. In any case the envelope conditions for $\tau^{\star}$ (the average marginal innovation's value equals the transfer) hold once innovations are pooled over the ironing region. 
This has the effect of shifting the high-quality type $\left(\sigma_{1}, m_{1}\right)$ to a higher $k$ (higher isoreward curve; note that $k=\sigma=m$ on the $45^{\circ}$ line, independently of $\alpha$ ) and the low-quality type $\left(\sigma_{2}, m_{2}\right)$ to a lower $k$. This in turn implies that for locally fixed transfers $\tau^{*}(k ; \alpha)$, a small increase in $\alpha$ at the margin will attract in marginal (cost equal to reward) high-quality innovations and crowd out marginal low-quality innovations.

The further a point is from the $(\sigma=m=k) 45^{\circ}$ line, the more quickly the $k$ value corresponding to the point increases (if the point is above the line), or decreases (if it is below), in $\alpha$. Just as the production of a Cobb-Douglas economy responds to a shift in shares at a rate proportional to the ratio of factors, the rate of moving up (or down) isoreward curves $\frac{d \sigma^{1-\alpha} m^{\alpha}}{d \alpha}$ for an innovation of partial type $x$ is proportional to $\log (x)$, because isoquants are log-linear: $\log (k)=(1-\alpha) \log (\sigma)+\alpha \log (m)$.

The value of innovations along any particular isoreward curve is proportional to $x^{1-2 \alpha}$ by Subsection III.A. The social benefit associated with raising $\alpha$ is that high $x$ innovations are created more frequently: To the extent that $x^{1-2 \alpha}$ covaries with $\log (x)$ (which it must to some extent), more beneficial innovations will be selected by higher values of $\alpha .10$ Thus, an increase in the covariance between the extra reward given to (marginal) innovations and the value of these innovations, $\operatorname{Cov}\left[x^{1-2 \alpha}, \log (x) \mid k, c=\tau^{\star}(k ; \alpha)\right]$, pushes towards greater market power. Furthermore, because $\lim _{\alpha \rightarrow \frac{1}{2}} \frac{x^{1-2 \alpha}-1}{1-2 \alpha}=\log (x)$, for $\alpha$ close to $\frac{1}{2}$,

$$
\operatorname{Cov}\left[x^{1-2 \alpha}, \log (x) \mid k, c=\tau^{\star}(k ; \alpha)\right] \approx(1-2 \alpha) \operatorname{Var}\left[\log x \mid k, c=\tau^{\star}(k ; 1)\right]
$$

Thus, if one is considering near-monopoly pricing rules, the incentive for marginally higher $\alpha$ is nearly proportional to the (conditional) variance of the logarithm of $x$, which, as noted in the introduction, is a standard measure of inequality. We therefore refer to $\operatorname{Var}(\log x)$ as the inequality of supply.

On the other hand, raising $\alpha$ reduces the value of all innovations that are created, not just the marginal ones (with $c=\tau^{\star}(k ; \alpha)$ ). Thus, the relative weight on this distortion is the ratio of the mass of infra-marginal innovations to the density of marginal innovations along a given

\footnotetext{
${ }^{10}$ See Veiga and Wevl (2012) for a general analysis of this logic of smooth screening.
} 
isoreward curve. Let

$$
\eta \equiv \frac{f_{c}\left(\tau^{\star}(k ; \alpha) \mid k\right) \tau^{\star}(k ; \alpha)}{F_{c}\left(\tau^{\star}(k ; \alpha) \mid k\right)}
$$

denote the elasticity of innovation supply with respect to reward, where $f_{c}$ and $F_{c}$ are the (conditional) marginal pdf and $\operatorname{cdf}$ of $c$, respectively. The inequality and elasticity of innovation supply can be formally shown to be the key determinants of the net marginal social benefit of ex-post distortion. Let $\tau^{\star^{\prime}}$ denote the derivative of $\tau$ with respect to $k$.

Corollary 1: $W^{\prime}(\alpha) \propto$

$$
E\left[k^{4}[\underbrace{(1-2 \alpha) \frac{\tau^{\star^{\prime}}}{k} \eta \operatorname{Cov}\left(\log x, \frac{x^{1-2 \alpha}-1}{1-2 \alpha}\right)}_{\text {sorting }}-\underbrace{\left.\frac{Q \alpha}{(1-\alpha)^{3} \epsilon^{\prime}} E\left(x^{1-2 \alpha}\right) E\left(x^{1-2 \alpha} \mid c<\tau^{\star}\right)\right]}_{\text {ex-post distortion }}],\right.
$$

where the outer expectation is taken over $k$, the covariance and inner expectation over $x$, all quantities inside the expectation are evaluated conditional on $k, c=\tau^{\star}(k ; \alpha)$ where not explicitly stated, and all expectations are taken under $\tilde{f}$. A necessary condition for the optimal choice of $\alpha$ is that $W^{\prime}(\alpha)=0$.

Proof. We provide the proof assuming continuous differentiability of $\tau^{\star}$ (the result however does not hinge on the differentiability of $\left.\tau^{*}\right)$.

$$
\begin{gathered}
\left.\frac{\partial}{\partial \alpha}\left[\int_{\sigma} \int_{m} \int_{c=0}^{\tau^{\star}\left(\sigma^{1-\alpha} m^{\alpha} ; \hat{\alpha}\right)}[\sigma m S(a(\alpha))-c] f(\sigma, m, c) d c d m d \sigma\right]\right|_{\alpha=\hat{\alpha}}= \\
\int_{\sigma} \int_{m}\left(\sigma^{1-\hat{\alpha}} m^{\hat{\alpha}} \tau^{\star^{\prime}} \log \left(\frac{m}{\sigma}\right)\left(\sigma m S-\tau^{\star}\right) f\left(\sigma, m, \tau^{\star}\right)-\int_{c=0}^{\tau^{\star}} \sigma m a^{\prime} Q \epsilon f(\sigma, m, c) d c\right) d m d \sigma
\end{gathered}
$$

where all variables are evaluated at $\hat{\alpha}$. Recalling that $\epsilon(a(\alpha))=\frac{\alpha}{1-\alpha}$, and thus, $a^{\prime}=\frac{1}{(1-\alpha)^{2} \epsilon^{\prime}}$, $W^{\prime}$ in the $(k, x)$ space can be rewritten as:

$$
\int_{k} \int_{x}\left[k \tau^{\star^{\prime}} \log (x)\left(k^{2} x^{1-2 \hat{\alpha}} S-\tau^{\star}\right) \tilde{f}\left(k, x, \tau^{\star}\right)-\int_{c=0}^{\tau^{\star}} k^{2} x^{1-2 \hat{\alpha}} \frac{\hat{\alpha} Q}{(1-\hat{\alpha})^{3} \epsilon^{\prime}} \tilde{f}(k, x, c) d c\right] d x d k .
$$

Letting $\tilde{f}_{k}(k)$ be the marginal distribution under $\tilde{f}$ of $k$ (and similarly with other subscripts 
of densities), this becomes

$$
\begin{aligned}
\int_{k} E_{x, \tilde{f}} & {\left[\left[k \tau^{\star^{\prime}} \log (x)\left(k^{2} x^{1-2 \hat{\alpha}} S-\tau^{\star}\right) \mid c=\tau^{\star}, k\right] \tilde{f}_{k}\left[\tau^{\star} ; \hat{\alpha}\right]\right.} \\
& \left.-k^{2} \frac{\hat{\alpha} Q}{(1-\hat{\alpha})^{3} \epsilon^{\prime}} E_{x, \tilde{f}}\left(x^{1-2 \hat{\alpha}} \mid c<\tau^{\star}, k\right) \tilde{F}_{k}\left(\tau^{\star} ; \hat{\alpha}\right)\right] \tilde{f}(k) d k .
\end{aligned}
$$

Because from equation (3),$\tau^{\star}=E_{x, \tilde{f}}\left(k^{2} x^{1-2 \hat{\alpha}} S \mid c=\tau^{\star}, k\right)$, we can rewrite the derivative as

$$
\begin{aligned}
\mathrm{S} \int_{k} k^{4} & {\left[\frac{\tau^{\star^{\prime}}}{k} \operatorname{Cov}_{x, \tilde{f}}\left(\log (x), x^{1-2 \hat{\alpha}}|| c=\tau^{\star}, k\right)\right.} \\
& \left.-\frac{\hat{\alpha} Q}{(1-\hat{\alpha})^{3} \epsilon^{\prime}} \frac{E_{x, \tilde{f}}\left(x^{1-2 \hat{\alpha}} \mid c<\tau^{\star}, k\right) E_{x, \tilde{f}}\left(x^{1-2 \hat{\alpha}} \mid c=\tau^{\star}, k\right)}{\eta\left(\tau^{\star} \mid k\right)}\right] \tilde{f}_{k c}\left(k, \tau^{\star}\right) d k
\end{aligned}
$$

where $\tilde{f}(k, c)$ is the marginal distribution of $(k, c)$. If we now normalize by dividing by $S \int_{k} \tilde{f}\left(k, \tau^{\star}\right) d k$, remove remaining unnecessary arguments and subscripts and multiply and divide the first term in the appropriate places by $1-2 \alpha$, we obtain the desired expression.

For the first-order condition to actually characterize the optimum, the problem must be (quasi-)concave. While we failed to find simple conditions on primitives to ensure this, we show in Appendix B that, so long as the product of the inequality and elasticity of innovation supply does not increase too rapidly with $\alpha$, especially for intermediate values of $\alpha$, the problem is concave. All of our computational simulations exhibit such concavity.

Intuitively, and as confirmed in Theorem 1 below, the distortion and screening effects should call for an optimal degree of market power $\alpha^{*}$ between ex-post efficiency $(\alpha=0)$ and monopoly pricing $(\alpha=1 / 2)$. Indeed $\alpha^{*}$ is strictly below $1 / 2$. Ensuring that $\alpha^{*}$ be strictly positive requires a very weak assumption. An example of a condition ensuring $\alpha^{*}>0$ is 11 No-shooting-in-the-dark condition. Offering an arbitrary, non-contingent prize $\tau$ results in negative welfare:

$$
\int_{\sigma} \int_{m} \int_{0}^{\tau}(\sigma m S(0)-c) f(\theta) d \theta<0
$$

To see why such a condition makes ex-post efficiency suboptimal, note that, using $S^{\prime}(0)=0$, the formula for $W^{\prime}$ in the proof of Corollary 1, and the fact that $S^{\prime} a^{\prime}=Q \varepsilon a^{\prime}=Q a^{\prime} \alpha /(1-\alpha)=0$

\footnotetext{
${ }^{11}$ The no-ironing condition in Appendix A also suffices.
} 
for $\alpha=0$,

$$
W^{\prime}(0)=\int_{\sigma} \sigma \tau^{* \prime}(\sigma ; 0)\left[\int_{m}\left[\sigma m S(0)-\tau^{*}(\sigma ; 0)\right] \log \left(\frac{m}{\sigma}\right) f\left(\sigma, m, \tau^{*}(\sigma ; 0)\right) d m\right] d \sigma
$$

Because

$$
\tau^{*}(\sigma ; 0)=\sigma S(0) E\left[m \mid c=\tau^{*}(\sigma ; 0)\right]
$$

the term in brackets is a covariance and is positive (as $m$ and $\log m$ covary positively). So $W^{\prime}(0)>0$ unless $\tau^{*}$ is constant (recall that $\tau^{*}$ must be non-decreasing in $\sigma$ due to the possibility of free disposal). But the no-shooting-in-the-dark condition rules out a non-quantitydependent prize.

Theorem 1: An optimal value of $\alpha, \alpha^{*}$, exists, and $0 \leq \alpha^{*}<1 / 2$. Furthermore, under the "no-shooting-in-the-dark" condition, $\alpha^{*}>0$.

Proof. See Online Appendix, Section 4.

\section{III.C. Approximation theorems}

Assuming quasi-concavity, we now consider the limit as the elasticity and inequality of innovation supply grow large or small.

Theorem 2 (Optimal near-monopoly pricing): Let $\pi \equiv \sigma m Q(1)$ be the monopoly profit associated with an innovation and $V_{1}$ be defined by:

$$
V_{1} \equiv \frac{E\left[\pi^{2} \operatorname{Var}\left(\log (x) \mid \pi, c=\frac{S(1)}{Q(1)} \pi\right) \eta\left(\frac{S(1)}{Q(1)} \pi \mid \pi\right)\right]}{E\left[\pi^{2}\right]}
$$

where the expectations are taken with respect to $\pi$ under the distribution over $(\pi, x, c)$ induced by $f(\sigma, m, c)$ and the variance is taken with respect to the conditional-on- $\pi$ distribution of $x .12$ Then, if $W$ is quasi-concave or if we consider only $\alpha$ values sufficiently near $\frac{1}{2}$, the optimal value of $\alpha$ is

$$
\alpha^{\star}=\frac{1}{2}-\frac{Q(1)}{S(1) \epsilon^{\prime}(1) V_{1}}+O\left(\frac{1}{V_{1}^{2}}\right)
$$

\footnotetext{
${ }^{12}$ Formally this is $\tilde{f}$ at $\alpha=\frac{1}{2}$.
} 
Thus for $V_{1}$ sufficiently large, $\alpha^{\star}$ approaches $\frac{1}{2}$; that is, as the value-weighted average of the product of the elasticity and inequality of innovation supply grow large, near-monopoly pricing becomes optimal.

Proof. By Corollary 1, rearranged slightly, the necessary condition for determining $\alpha^{\star}$ is $(1-2 \alpha) S E\left[\frac{1}{S} \tau^{\star^{\prime}} k^{3} \eta \operatorname{Cov}\left(\log (x), \frac{x^{1-2 \alpha}-1}{1-2 \alpha}\right)\right]=\frac{Q \alpha}{2(1-\alpha)^{3} \epsilon^{\prime}} E\left[k^{4} E\left(x^{1-2 \alpha}\right) E\left(x^{1-2 \alpha} \mid c<\tau^{\star}\right)\right]$.

Letting $\omega(\alpha) \equiv(1-2 \alpha) \frac{S(1-\alpha)^{3} \epsilon^{\prime}}{Q \alpha}$ and

$$
\beta(\alpha) \equiv \frac{E\left[\frac{1}{S} \tau^{\star^{\prime}} k^{3} \eta \operatorname{Cov}\left(\log (x), \frac{x^{1-2 \alpha}-1}{1-2 \alpha}\right)\right]}{V_{1} E\left[k^{4} E\left(x^{1-2 \alpha}\right) E\left(x^{1-2 \alpha} \mid c<\tau^{\star}\right)\right]},
$$

the first-order condition becomes $\omega(\alpha) \beta(\alpha) \equiv \kappa(\alpha)=\frac{1}{V_{1}}$. Proposition 2 in Appendix $\mathrm{B}$ shows that $\kappa$ is locally decreasing near $\alpha=\frac{1}{2}$ and thus we may consider the local inversion of $\kappa$ in this neighborhood $\kappa_{\delta_{\frac{1}{2}}}^{-1}$. Note that $\omega\left(\frac{1}{2}\right)=0$. To determine $\beta\left(\frac{1}{2}\right)$ note first that $\tau^{\star}\left(k ; \frac{1}{2}\right)=$ $S(1) k^{2}$ by equation (3) and thus $\tau^{\star^{\prime}}\left(k ; \frac{1}{2}\right)=2 S(1) k$. Second, note that by L'Hôpital's rule, $\lim _{\alpha \rightarrow \frac{1}{2}} \frac{x^{1-2 \alpha}-1}{1-2 \alpha}=\log (x)$ so $\lim _{\alpha \rightarrow \frac{1}{2}} \operatorname{Cov}\left(\log (x), \frac{x^{1-2 \alpha}-1}{1-2 \alpha}\right)=\operatorname{Var}(\log (x))$. Third, note that when $a=1, k^{2} Q(1)=\pi$ and that $E\left(x^{1-2 \alpha}\right)=E\left(x^{1-2 \alpha} \mid c<\tau^{\star}\right)=1$. Thus $\lim _{\alpha \rightarrow \frac{1}{2}} \beta(\alpha)=2$. Thus Taylor expanding $\kappa_{\delta_{\frac{1}{2}}}^{-1}$ about 0 yields

$$
\alpha^{\star}=\frac{1}{2}+\kappa_{\delta_{\frac{1}{2}}}^{-1^{\prime}}(0) \frac{1}{V_{1}}+O\left(\frac{1}{V_{1}^{2}}\right)
$$

By the inverse function theorem,

$$
\kappa_{\frac{1}{2}}^{-1^{\prime}}(0)=\frac{1}{\kappa^{\prime}\left(\frac{1}{2}\right)}=\frac{1}{\omega^{\prime}\left(\frac{1}{2}\right) \beta\left(\frac{1}{2}\right)}=\frac{1}{-2 \frac{S(1)\left(1-\frac{1}{2}\right)^{3} \epsilon^{\prime}(1)}{Q(1) \cdot \frac{1}{2}} \cdot 2}=-\frac{Q(1)}{S(1) \epsilon^{\prime}(1)}
$$

so that rearranging equation (66) yields the result.

Intuitively, as the inequality and elasticity of innovation supply grow, the screening benefit grows and monopoly pricing becomes optimal in the limit. In this limit many of the complexities above disappear: isoreward curves become isoprofit sets, optimal transfers collapse to the 
social surplus of every innovation, namely $\frac{S(1)}{Q(1)}$ times profit, and optimal pricing is monopoly pricing.

This particular approximation is also of interest for a pragmatic reason. Most advanced capitalist nations use primarily market-power-based schemes for rewarding innovation: thus current policy has $\alpha$ near $\frac{1}{2}$. Hence a local approximation to optimal policy near this point seems most useful for a gradualist interested in moving cautiously away from this point. If $V_{1}$ is not in fact measured to be large, one may only conclude it should move down significantly, but how far exactly is not clear as the approximation becomes poor. After policy has been changed, the model may be recalibrated and further adjustments enacted, in the spirit of Chetty (2009).

To illustrate the generality of the approach, we also consider an approximation about $\alpha=0$, providing a partial converse to Theorem 2 ,

Theorem 3 (Partial converse): Let $V_{0}$ be defined by

$$
V_{0} \equiv \frac{E\left[\sigma^{4} \frac{\log \left(\tau_{0}\right)^{\prime} \operatorname{Cov}\left(\log (m), m \mid \sigma, c=\tau_{0}(\sigma)\right) \eta\left(\tau_{0}(\sigma) \mid \sigma\right)}{\sigma E\left(m \mid \sigma, c<\tau_{0}(\sigma)\right)}\right]}{E\left(\sigma^{4}\right)}
$$

where $\tau_{0}$, the optimal reward schedule at $\alpha=0$, is defined implicitly by $\tau_{0}(\sigma)=\sigma S(0) E\left(m \mid c=\tau_{0}(\sigma), \sigma\right)$, the outer expectation is taken with respect to $\sigma$ and the inner expectation and covariance are taken over $m$, all under the measure $f$. Then, if $W$ is quasi-concave in $\alpha$ or if we consider only $\alpha$ values sufficiently near 0 , the optimal value of $\alpha$ is

$$
\alpha^{\star}=\epsilon^{\prime}(0) V_{0}+O\left(V_{0}^{2}\right)
$$

Thus for $V_{0}$ sufficiently small, $\alpha^{\star}$ approaches 0 .

Proof. Follows mutatis mutandis from analogous logic in the proof of Theorem 2 (the Taylor expansion is now about 0 ), as in this limit $k=\sigma, x^{1-2 \alpha}=x$ when $\alpha=0$ and $\left.\frac{Q}{(1-\alpha)^{3} \epsilon^{\prime}}\right|_{\alpha=0}=$ $\frac{1}{\epsilon^{\prime}(0)}$.

Intuitively, as $m$ becomes perfectly known or all innovations become infra-marginal, the screening benefit of ex-post distortion becomes small. Optimal policy becomes ex-post-efficient 
pricing coupled with demand-dependent prizes.

Numerical simulations verify the validity of our intuitions between these two polar cases. For example, we considered a simulation in which $\pi$ is drawn from a uniform distribution, $x$ is drawn log-normally and $c$ is drawn exponentially (so that its elasticity can be unambiguously adjusted), all independent of one another. Increasing the log-variance of $x$ increases $a^{\star} \equiv a\left(\alpha^{\star}\right)$ through the full range of values. Furthermore, as the elasticity of innovation supply increases, so too does $a^{\star}$, again through the full range of values 13

\section{III.D. Beyond the stretch parameterization}

An important assumption of the analysis above is that the demand function is given by a stretch parameterization. This parameterization is useful because it implies proportionality between average consumer surplus and profit for all products that charge the same fraction of their monopoly price. Crucially in our final expression, Equation (5), the ratio $\frac{S(1)}{Q(1)}$ of social surplus to monopoly profit separates multiplicatively from the $V_{1}$ variance expression on which we focus 14

Suppose, instead, that this ratio may differ across products. Then the value of a product when price is close to the monopoly level is proportional, along a given $\alpha$ isoreward curve, to $\frac{S^{\star}}{\pi^{\star}} x^{1-2 \alpha}$, where $\frac{S^{\star}}{\pi^{\star}}$ is the ratio of social surplus to monopoly profit at the monopoly optimum and $x=\frac{p}{q}$. The marginal reward to innovators when $\alpha$ increases is still proportional to $\log (x)$. Thus the sorting value along the $k=p^{\alpha} q^{1-\alpha}$ isoreward curve for $\alpha$ close to $\frac{1}{2}$ is proportional to

$$
\begin{aligned}
& \operatorname{Cov}\left[\log (x), \frac{S^{\star}}{\pi^{\star}} \frac{x^{1-2 \alpha}-1}{1-2 \alpha} \mid k=p^{\alpha} q^{1-\alpha}\right] \rightarrow \operatorname{Cov}\left[\log (x), \frac{S^{\star}}{\pi^{\star}} \log (x) \mid k=p q\right]= \\
& E\left[(\log (x))^{2} \frac{S^{\star}}{\pi^{\star}} \mid \pi=k\right]-E[\log (x) \mid \pi=k] E\left[\frac{S^{\star}}{\pi^{\star}} \log (x) \mid \pi=k\right] .
\end{aligned}
$$

Now suppose that $\frac{S^{\star}}{\pi^{\star}}$ is independent of $x$ along any isoprofit curve. Then we can separate $E\left[\frac{S^{\star}}{\pi^{\star}} \mid \pi=k\right]$ from both terms of the expression and obtain the same result replacing $\frac{S(1)}{Q(1)}$

\footnotetext{
${ }^{13}$ Python code for our simulations is available at http://www.glenweyl.com.

${ }^{14}$ Similar logic follows away from the near-monopoly limit. See Veiga and Weyl (2012) for a more general development of this covariance logic.
} 
with $E\left[\frac{S^{\star}}{\pi^{\star}} \mid \pi=k\right] 15$ Thus, given that $\frac{S(1)}{Q(1)}$ plays little role in our theory and thus is, anyway, best interpreted as an average, we would argue that the core assumption of our model is not so much the stretch parameterization as the (iso-profit-conditional) independence of $\frac{S^{\star}}{\pi^{\star}}$ from $x=\frac{p}{q}$ embedded in it.

While we believe this independence assumption is a good approximation in many cases, it is useful to consider what happens when it is violated. First, suppose that $x$ is positively related to $\frac{S^{\star}}{\pi^{\star}}$; that is, among products earning the same profit, those with high prices and small quantities have more convex demand curves and thus more consumer surplus per unit of profit (Weyl and Fabinger, 2011). Then our formula underestimates the benefits of market power and it may be optimal to have prices above monopoly optimal. A story justifying this possibility is that higher-price, low-volume goods are typically bought by the wealthy, among whom the income distribution is known to be more convex (Piketty and Saez, 2003) 16 On the other hand, if within an iso-profit set, mass-market, low-mark-up products have more convex demand, then our formula overestimates the sorting benefits and thus the social value of market power. We think that this latter case is implausible in most applications and thus that if anything our results are likely to understate the benefits of market power.

\section{Applications And Implementation}

This section discusses the relevance and implementation of our mechanism in three contexts: government rewards for innovation, rewards by platforms to developers of applications, and the procurement of public infrastructure. While our mechanism may appear somewhat abstract, we use this section to show how it is, in fact, similar to many existing institutions and represents

\footnotetext{
${ }^{15}$ We would also need to similarly replace $\epsilon^{\prime}(1)$ with $E\left[\left(\epsilon^{\prime}\right)^{\star} \mid \pi=k\right]$ where $\left(\epsilon^{\prime}\right)^{\star}$ is the average semi-elasticity $\left(\frac{p d \epsilon}{d p}\right)$ of the elasticity of demand with respect to price at the monopoly price. Note that the derivative here is taken with respect to $p$ rather than $a$ as demand curves are no longer drawn from a class of demand curves linked by a stretch parameterization; the use of the semi-elasticity provides the equivalent normalization. It can easily be shown, as in Weingarten (2011), that $\left(\epsilon^{\prime}\right)^{\star}=\frac{1}{\rho}$ where $\rho$ is the rate a which the monopolist passes through a local increase in a specific tax to prices. Weyl and Fabinger (2011) show that $\frac{S^{\star}}{\pi^{\star}}=1+\bar{\rho}$, where $\bar{\rho}$ is the quantity-weighted average of pass-through over tax rates above 0 .

${ }^{16}$ In particular, we believe this because the distribution of income among middle and lower-income groups is less convex (following a log-normal distribution rather than a Pareto distribution) than among the wealthy and because most products reaching a mass market are pitched to the "lowest common denominator," having few individuals who value the product far more than a typical individual.
} 
a continuous, rather than radical, departure from these.

\section{IV.A. Public innovation incentives}

The bulk of our paper is formulated in terms of government-granted incentives for innovation by private firms, such as patents and prizes. We therefore begin with this application.

A simple application of our results is to the relative attractiveness of prizes compared to IP in the development of new medicines compared to software and other high-technology products. Kremer, his co-authors and many others have advocated prizes for the development of new medicines. More broadly, many developed countries have extensive price controls, centralized bargaining, subsidies and other non-market mechanisms for increasing access to medicines 17 As in a prize system, these determine rewards per unit sold independently of the discipline of market prices. On the other hand, most advanced economies use, and most economists would advocate, market-oriented pricing of high technology products, from software to tablet computers.

These intuitions square with our reasoning because, at least in many cases, the perconsumer value of medicines can be judged somewhat objectively using clinical trials and standard calculations of the monetary value of added quality-adjusted life years. Thus the innovator has little private information on $m$ ex post. Conversely high technology exhibits nearly the opposite pattern: it is typically far from apparent how much consumers value various new electronics and software until the market demonstrates this. Taking our example from the introduction, many engineers believed AOL would be worth little compared to Netscape because they failed to take into account AOL's relative ease and social benefits for non-specialists.

Let us now turn to the implementation of the optimal scheme. A study committee would use data and experience to estimate, based on ex-ante observable product characteristics, the optimal value of $\alpha$ and the transfers assigned to each isoreward curve. Suppose that, based on an empirical calibration it were determined that for some relevant industry or category of innovative products $\alpha^{\star} \approx .45$ and that social value is approximately linear in $p^{81} q^{1}=$

\footnotetext{
${ }^{17}$ These policies have traditionally been explained by the government discounting innovator surplus and/or low $\eta$ at the country level, so that in the absence of cross-country coordination, each country internalizes too little of its effect on innovation supply. However, the difference in policy treatment between the medical and other sectors suggests a role for private information about quality.
} 
$\left(p^{.45} q^{.55}\right)^{1.8}$, with a constant of proportionality of 1.5 . Because $\alpha^{\star}$ is relatively close to .5 , our local approximation is plausible. [If we were to find a significantly lower $\alpha^{\star}$, we would likely advocate moving $\alpha$ gradually so as to allow an empirical recalibration prior to further adjustments.]

In this case, our approximated optimal transfer would be $1.5 p^{81} q$. To implement this, a natural approach would be to allow the entrepreneur to keep her full profit, $p q$, and then, in addition to that, provide her with a specific (per-unit) subsidy on the good that depends on its price, as well as a $50 \%$ subsidy on profit. The net transfers of the subsidy would need to be $1.5 p^{81} q-1.5 p q=\left(p^{81}-p\right) 1.5 q$. While this still seems a bit dauntingly nonlinear, the scheme can be well-approximated by a per-unit subsidy depending linearly on price 18

Admittedly, implementing this scheme relies on the ability of the public authority to observe the price and quantity of the products sold. Such observations might be difficult and subject to fraud or manipulation. However, the required observations are to a large extent already a part of the work of the government in its capacity as collector of sales and corporate income taxes. In fact, many policies used to stimulate or reduce the prices of new innovations rely on such detailed observations, such as subsidies for the uptake of green technology (which requires observing quantities) and price controls on drugs (which require observing prices). Other policies used for innovation incentives, such as research and development (R\&D) subsidies, require arguably even less-accessible information on $R \& D$ expenditures by firms. As with the other policies, potential manipulation can be partly deterred by the vigorous enforcement of anti-fraud statutes.

\section{IV.B. Platforms}

A two-sided platform, such as an operating system, must attract multiple sides of a market, say end-users and application developers. A key decision such platforms face is how to "regulate" the relationship between application developers and end-users. Should it let application

\footnotetext{
${ }^{18}$ One simple approximation would be to give a per-unit net subsidy per-unit of $2.5-.6 p$. This never differs from the optimal scheme by more than $10 \%$ if prices are between $\$ 10$ and $\$ 100$ and never by more than a $\$ 1.50$ per unit over this range. Other rules and other approximations would be relevant for other products and thus the government would like want to develop a computerized methodology allowing firms to determine the value they are likely to receive in various scenarios.
} 
developer $i$ charge a monopoly price $\left(p_{i}=m_{i}\right)$ for his application, effectively giving him IP? Or should it bundle the application, free to the consumers, with the platform, paying the developer a prize-like up-front fee:19

The analogy between a platform and a social planner can be made more formal and is even exact when end-users differ in an idiosyncratic parameter of taste for the platform and there is a large number of applications. Let $i \in[0,1]$ denote the set of potential innovations, $\delta_{i}=1$ if the innovation is developed and $\delta_{i}=0$ otherwise. Consumers are indexed by $k$, uniform in $[0,1]$. Consumer $k$ 's net payoff is $\int_{0}^{1} V_{i}\left(p_{i} ; \sigma_{i}, m_{i}\right) \delta_{i} d i-T-\xi_{k}$, where $\xi_{k}$ is distributed according to $H(\cdot)$. Application developer $i$ introduces the innovation if and only if $t_{i} \geq c_{i}$. The platform's profit is then $\left[T+\int_{0}^{1}\left[\pi_{i}\left(p_{i} ; \sigma_{i}, m_{i}\right)-t_{i}\right] \delta_{i} d i\right]\left[1-H\left(t-\int_{0}^{1} V_{i}\left(p_{i} ; \sigma_{i}, m_{i}\right) \delta_{i} d i\right)\right]$, or after a change in variables and using $S_{i} \equiv V_{i}+\pi_{i},\left[\widehat{T}+\int_{0}^{1}\left[S_{i}\left(p_{i} ; \sigma_{i}, m_{i}\right)-t_{i}\right] \delta_{i} d i\right][1-H(\widehat{T})]$. Thus the platform first maximizes $\int_{0}^{1}\left[S_{i}\left(p_{i} ; \sigma_{i}, m_{i}\right)-t_{i}\right] \delta_{i} d i$ and then chooses the surplusadjusted price $\widehat{T}$.

The platform aims at maximizing total surplus (end-users' gross surplus) minus the rewards given to developers. Therefore, using the straightforward extension of the paper's analysis to the case where the social planner cares nothing for the entrepreneur, included in a previous version of this paper (Weyl and Tirole, 2011), our analysis carries through without any modification.

Online music stores, such as Apple's iTunes store and Rhapsody, use price caps, fixed or proportional licensing fees and low (or purely bundled) prices to consumers. In our model, this is rationalized by the fact that only a small fraction of the total revenue for a song comes from any of these individual stores and thus the elasticity of innovation supply with respect to a change in their revenue from one of these stores is small. Contrast this with the Apple App store, which allows total pricing freedom to App developers and gives them a share of revenue. Given that applications are developed almost exclusively for the iPhone or another individual platform, the elasticity of innovation supply is likely much higher and thus there is more burden on Apple to sort out which applications deserve greater rewards by using market power. Additionally, the heterogeneity of $m$ seems likely to be higher in the app than music

\footnotetext{
${ }^{19}$ A similar tradeoff arises when the "application developer" is an internal division and the platform wants to provide it with incentives to develop useful innovations.
} 
market as even in the open market, prices differ relatively little for most singles or albums, while Weingarten (2011) finds a large variance in price and market size across applications earning the same profits. Thus our model provides a potential rationalization of observed patterns of platform design in these media markets.

Implementation of the optimal scheme is particularly plausible in the case of a platform. For example, Apple collects all revenues from products sold on its store and transmits a part $(70 \%$ currently) of them to the application developers. Apple could easily make this payment a nonlinear function of the price charged and quantity sold. In fact, Weingarten (2011) combines our analysis with data from the App Store to calibrate an optimal policy. He finds that the optimal scheme, while close to that currently used by Apple, would involve some discouragement of high prices $\left(\alpha^{\star} \approx .44\right)$, which both seems natural given Apple's policies on iTunes and would require only small changes to their current contracts. Again this direct payment version could easily be approximately implemented using piecewise linear contracts if this were easier for developers to understand.

\section{IV.C. Public infrastructure}

The traditional approach to building a new highway or new train tracks is to enter a procurement contract with an infrastructure builder, and then to turn to a separate infrastructure operator to manage it; the infrastructure may then be accessed at a relatively low price. By contrast, under a public-private partnership (PPP), the builder of the new infrastructure derives substantial revenue from its later operations. Such a long-term approach links builder compensation to actual revenue derived from the end-user and is often vaunted as a way to screen out white elephants.

Purely public projects may be seen in a similar light if we consider the limiting case of observed costs and a political entrepreneur seeking funding from the public purse for a project, motivated to have a successful project by the prestige, career enhancement or other non-pecuniary benefit it brings. In Online Appendix Section 4 we show that in this case an optimal scheme (the optimal scheme if transfers are - at least slightly - socially costly) is to reimburse the known cost provided that the innovation satisfy a minimum scoring rule: 
$q^{1-\alpha} p^{\alpha} \geq k$, and nothing if this score is not reached. This offers a simple implementation of our scheme in the context of public procurement. Additional incentives (perhaps necessary because of uncertainty and risk aversion) could be provided by giving awards, public praise or career advancement to bureaucrats whose schemes achieve a high score.

When $\alpha$ is close to $\frac{1}{2}$, this minimum score is equivalent to a minimum profit level for the project. On the other hand, maximizing score is equivalent to maximizing a convex combination of consumer surplus and profits: $\lambda C S(p)+(1-\lambda) p D(p)$ where $\lambda=\frac{1-2 \alpha}{2-3 \alpha} 20$ Thus we may interpret policy regimes under which politicians or bureaucrats are under more pressure to recover costs by charging higher prices as high $\alpha$ regimes, and ones under which the emphasis is on consumer surplus and subsidies are required as corresponding to low $\alpha$. The role of the inequality and elasticity (now in the threshold level rather than reward space) of innovation supply is unchanged.

\section{Conclusion}

This paper aspires to make three contributions. First, in terms of modeling, we propose the intuitive stretch parameterization of demand to allow a smooth trade-off between quantitydependent prizes and IP. Second, on a technical level, we develop a simple isoreward approach to analyzing multidimensional screening problems: parameterize policies based on the shape of the isoreward curves they create and then solve separately for the structure and level of rewards using the envelope theorem. Finally, and substantively, we show how the inequality and elasticity of innovation supply are tightly connected to the optimality of market power as a reward for innovation, making precise and empirically testable the conjectures of classical thinkers.

Needless to say, our framework requires further elaboration in order to help fashion policy. Furthermore, given the foundational role that many of the issues we address in this paper play in several areas of price theory, we believe that our work opens a number of promising

\footnotetext{
${ }^{20}$ Taking the derivative of the objective function yields
}

$$
-\lambda D(p)+(1-\lambda) D(p)+(1-\lambda) p D^{\prime}(p) .
$$

Rearranging yields first-order condition $\frac{1-2 \lambda}{1-\lambda}=\epsilon$ and solving $\frac{1-2 \lambda}{1-\lambda}=\frac{\alpha}{1-\alpha}$ yields the result in the text. 
directions for future research. First, our general formula ought to be calibrated in specific environments beyond Weingarten's smartphone application. Second, several extensions would test the robustness of our insights: general analysis of demand uncertainty (under entrepreneur limited liability or risk aversion), as well as richer private information (duration, marketing, price discrimination, marginal costs) and richer instruments for screening demand would be welcome. By "richer instruments," we refer to the use of multiple price observations to obtain a better estimate of the demand function: intertemporal price variations, random schemes and geographic price discrimination. In Weyl and Tirole (2011), we analyzed such use of multiple price observations for very specific demand functions and showed that for these functions, the general message, in the form of a need to use "sufficient" market power "often enough" in order to screen in good innovations and out bad ones, still holds; while suggestive, these examples made use of rather special demand curves and therefore the robustness of the conclusions still needs to be investigated.

Finally, the extension of our techniques to accommodate R\&D races, licensing competition, imitation and cumulative innovation stands high on the research agenda. For instance, our techniques are likely to be helpful in analyzing the validity of the notion (central to antitrust doctrine) that acquired market power may be maintained but should not be extended. The acceptability of vertical foreclosure practices is often felt to depend on the extent of innovation/investment, while market power gained through horizontal mergers and predation are frowned upon in the absence of substantial efficiency gains. Formal analyses would be useful to help guide policy in these matters.

UNIVERSITY OF CHICAGO AND TOULOUSE SCHOOL OF ECONOMICS

TOULOUSE SCHOOL OF ECONOMICS 


\section{Appendices}

\section{A. Supply, Demand AND optimal transfers}

This appendix solves for the monotonicity-relaxed optimal $\tau^{\star \star}(k ; \alpha)$ function and conditions under which this function is, in fact, monotone. We can then rewrite the social planner's problem, with the substitution of $f$ into $\widetilde{f}$ (see Section III.A) and by switching the order of integration, as

$$
\max _{\tau(\cdot ; \alpha)} \int_{k} \int_{c=0}^{\tau(k ; \alpha)} \int_{x}\left(k^{2} x^{1-2 \alpha} S(a(\alpha))-c\right) \tilde{f}(k, x, c ; \alpha) d x d c d k \text { s.t. } \tau \text { increasing. }
$$

Consider the marginal cumulative distribution of innovations in terms of their cost of creation $c$, integrating out over $x$, lying along a particular isoreward curve, $F(\tau ; k, a) \equiv \frac{\int_{c=0}^{\tau} \int_{x} \tilde{f}(k, x, c ; \tilde{\alpha}) d x d c}{\int_{c=0}^{\infty} \int_{x} \tilde{f}(k, x, c ; \tilde{\alpha}) d x d c}$. This is the fraction of innovations that will be created if a reward $\tau$ is offered along this curve. $\Sigma(r ; k, \alpha) \equiv F^{-1}(r ; k, \alpha)$ is then the (clearly increasing) inverse supply of innovations lying along isoreward curve $k$, namely the reward necessary to induce a fraction $r$ of innovations lying along that curve to be created.

We can similarly define the social inverse demand for innovations. First, let us define the average value of an innovation lying on isoreward curve $k$ with cost $c$ by

$$
\bar{S}(c ; k, \alpha) \equiv k^{2} S(a(\alpha)) E_{x, \tilde{f}}\left[x^{1-2 \alpha} \mid k, c\right] \equiv k^{2} S(a(\alpha)) \cdot \frac{\int_{x} x^{1-2 \alpha} \tilde{f}(k, x, c ; \alpha) d x}{\int_{x} \tilde{f}(k, x, c ; \alpha) d x}
$$

Then $D(r ; k, \alpha) \equiv \bar{S}(\Sigma(r ; k, \alpha) ; k, \alpha)$ is the average value of a marginal innovation lying along isoreward curve $k$, given that a fraction $r$ of innovations lying on that isoreward curve have been created.

The optimal reward along the isoreward curve is the intersection of the supply and demand curves for innovations, assuming these intersect only once. To the extent that $\alpha<\frac{1}{2}$, $D$ will slope downwards if $x$ varies negatively with $c$ given $k$ and upwards in the reverse case, in a sense made more rigorous below. Both effects are dampened for large $\alpha$. So long as $D$ does not increase too quickly, supply and demand will have a unique intersection corresponding to the optimal quantity of innovations and reward along $k$ given $\alpha$. These optimal rewards ignore the monotonicity constraint that higher- $k$ isoreward curves must receive higher rewards. When $\alpha$ is relatively low, if $k$ is sufficiently negatively affiliated with $x$ given $c$, the optimal reward unconstrained by monotonicity may be decreasing in $k$; ruling out such strong negative affiliation ensures that the relaxed solution is in fact optimal.

Proposition 1: Suppose that for all $k, c$ and a fixed $\alpha$,

$$
\operatorname{Cov}_{x, \tilde{f}}\left[x^{1-2 \alpha}, \frac{\partial \log (f)}{\partial c} \mid k, c\right] \leq \frac{1}{k^{2} S(a)}
$$

and

$$
2 E_{x, \tilde{f}}\left[x^{1-2 \alpha} \mid k, c\right] \geq-k \operatorname{Cov}_{x, \tilde{f}}\left[x^{1-2 \alpha}, \frac{\partial \log (\tilde{f})}{\partial k} \mid k, c\right]
$$

Then the optimal reward function $\tau^{\star}(k ; \alpha)$, given $\alpha$, is defined for each $k$ by the unique value at which $D(\cdot ; k, \alpha)$ and $S(\cdot ; k, \alpha)$ intersect if $D(0 ; k, \alpha)>S(0 ; k, \alpha), D(1 ; k, \alpha)<S(1 ; k, \alpha)$ for 
all $k, a$ or by the appropriate boundary solutions otherwise.

These conditions are intuitive extensions of the classic Mirrlees (1979)-Rogerson (1985) monotone likelihood ratio property that ensures validity of first-order approaches in classical, single-dimensional screening problems. If as $x$ increases $\frac{\partial \log (f)}{\partial c}$ also increases, this exactly represents $x$ having a strong monotone likelihood ratio relationship with $c$. Thus, condition (10) can be viewed as stating that $c$ and $x$ are not "too" affiliated (Milgrom and Weber, 1982), while condition (11) can be seen as stating that $x$ is not too negatively affiliated with $k$. Note that ironing-free approach is always valid for sufficiently high values of $\alpha . \tau^{\star \star}(k ; \alpha)$ is monotone increasing for high values of $\alpha$, but must be ironed for low values of $\alpha$.

Proof. The first-order condition for the monotonicity-unconstrained optimum is exactly that $\bar{S}(\tau ; k, \alpha)=\tau$ for each $k$. This condition is sufficient for the monotonicity-unconstrained optimum if the first-order derivative with respect to $t, \bar{S}-\tau$, is monotone decreasing in $\tau$ (Guesnerie and Laffont, 1984). Because these expressions are clearly differentiable, given the smoothness assumed, this is equivalent to

$$
\frac{\partial \bar{S}}{\partial c} \leq 1 \Longleftrightarrow k^{2} S(a) \frac{\partial E_{x, \tilde{f}}\left[x^{1-2 \alpha} \mid k, c\right]}{\partial c} \leq 1 \Longleftrightarrow \operatorname{Cov}_{x, \tilde{f}}\left[x^{1-2 \alpha}, \frac{\partial \log (\tilde{f})}{\partial c}\right] \leq \frac{1}{k^{2} S(a)}
$$

Because $\tilde{f}$ differs from $f$ as a function of $c$ only by a multiplicative factor, we can replace $\tilde{f}$ with $f$ and obtain inequality (10) in the proposition. Furthermore an upward shift in $\bar{V}$ must then increase this optimal transfer (Milgrom and Shannon, 1994). Thus we are guaranteed that $\tau^{\star \star}$ will be monotone so long as $\bar{V}$ increases in $k$. Again by differentiability this is equivalent to (taking logs)

$$
\frac{\partial \bar{S}}{\partial k} \geq 0 \Longleftrightarrow \frac{2}{k}+\frac{\operatorname{Cov}_{x, \tilde{f}}\left[x^{1-2 \alpha}, \frac{\partial \log (\tilde{f})}{\partial k} \mid k, c\right]}{E_{x, \tilde{f}}\left[x^{1-2 \alpha} \mid k, c\right]} \geq 0
$$

which simplifies to condition (11). Thus if this condition also holds the $\tau^{\star \star}$ derived from supply and demand is in fact $\tau^{\star}$.

A (grossly) sufficient condition to ensure that when $\alpha=0, \tau^{\star}$ is not flat and therefore that $\alpha^{\star} \in\left(0, \frac{1}{2}\right)$ is obedience of inequality (11) when $\alpha=0$. At $\alpha=0$ this condition simplifies to

(at each $(\sigma, c)), 2 E_{m, f}[m \mid \sigma, c] \geq-\sigma \operatorname{Cov}_{m, f}\left[m, \frac{\partial \log (f)}{\partial \sigma} \mid \sigma, c\right]$. That is, $\sigma$ is not too negatively affiliated with $m$.

\section{B. SECOND-ORDER CONDITIONS FOR OPTIMAL MARKET POWER}

The first-order condition, that expression (4) is equal to 0 , is necessary but not sufficient for the socially optimal choice of $\alpha$. Some condition, such as quasi-concavity of $W$, is needed to ensure it selects even a local, much less a global, maximum. As in the Mirrlees problem, interpretable conditions on primitives to ensure this seem challenging to derive.

However, note that by Proposition 1 we know that the optimal value of $\alpha$ must be in the interior of the half-unit interval and thus $W^{\prime}(\alpha)$ must be eventually negative as $\alpha$ goes to 1 and eventually positive as $\alpha$ goes to 0 . While this certainly does not preclude several local minima or maxima in the interior, it does ensure that in the limiting cases of Subsection III.C. 
the optimal points picked out are at least local maxima. Computational simulations suggest that global quasi-concavity is common; the following proposition provides a standard sufficient condition for quasi-concavity, applied to this context.

\section{Proposition 2: Let}

$$
V(\alpha) \equiv \frac{E_{k, \tilde{f}}\left[k^{3} \tau^{\star^{\prime}}(k ; \alpha) \operatorname{Cov}_{x, \tilde{f}}\left(\log (x), \frac{x^{1-2 \alpha}-1}{1-2 \alpha}\right)\right]}{E_{k, \tilde{f}}\left[\frac{E_{x, \tilde{f}}\left(x^{1-2 \alpha} \mid c<\tau^{\star}(k ; \alpha), k\right) E_{x, \tilde{f}}\left(x^{1-2 \alpha} \mid c=\tau^{\star}(k ; \alpha), k\right)}{\eta\left(\tau^{\star}(k ; \alpha) ; k, \alpha\right)}\right]},
$$

where $\tau^{\star}$ is defined implicitly by equation (3) and can be solved for explicitly given $\alpha$ by the techniques of Appendix $A$ and footnote 9 .

If $\tau^{\star}$ is differentiable, $W$ is quasi-concave if for all $\alpha \in\left(0, \frac{1}{2}\right)$,

$$
\frac{d \log (V)}{d \alpha}<\frac{1+3 \alpha-6 \alpha^{2}+3 \alpha^{3}}{(1-2 \alpha)(1-\alpha)^{2} \alpha}+\frac{\alpha-(1-\alpha) a(\alpha) \epsilon^{\prime \prime}(a(\alpha))}{\epsilon^{\prime}(a(\alpha))(1-\alpha)^{3}}
$$

This condition always holds for $\alpha$ sufficiently close to either 0 or $\frac{1}{2}$.

Proof. The first-order derivative from expression (44) is exactly

$$
(1-2 \alpha) V(\alpha)-\frac{Q \alpha}{(1-\alpha)^{3} \epsilon^{\prime}}
$$

Given that all terms here are strictly positive for $\alpha \leq \frac{1}{2}$, using the standard ratio monotonicity condition, a sufficient condition for quasi-concavity is that $\frac{(1-2 \alpha)(1-\alpha)^{3} \epsilon^{\prime}(a(\alpha))}{Q(a(\alpha))} V(\alpha)$ or its $\log$ is declining in $\alpha$ (recall that $\left.a^{\prime}(\alpha)=\frac{1}{(1-\alpha)^{2} \epsilon^{\prime}}\right)$ :

$$
\frac{d \log (V)}{d \alpha}<\frac{3}{1-\alpha}+\frac{2}{1-2 \alpha}+\frac{1}{\alpha}-\frac{\epsilon^{\prime \prime}}{\epsilon^{\prime}(1-\alpha)^{2}}+\frac{\alpha}{\epsilon^{\prime} a(1-\alpha)^{3}}=\frac{3(1-2 \alpha) \alpha+2(1-\alpha) \alpha+(1-\alpha)(1-2 \alpha)}{(1-\alpha)(1-2 \alpha) \alpha}+\frac{\alpha-\epsilon^{\prime \prime} a(1-\alpha)}{\epsilon^{\prime} a(1-\alpha)^{3}}
$$

which gives the desired inequality by expansion and simplification.

Note that by differentiability and strictly declining marginal revenue

$$
\lim _{a \rightarrow 0} \epsilon^{\prime}(a), \lim _{a \rightarrow 1} \epsilon^{\prime}(a)>0
$$

and that $\lim _{\alpha \rightarrow 0} 1+3 \alpha-6 \alpha^{2}+3 \alpha^{3}=1$ and $\lim _{\alpha \rightarrow \frac{1}{2}} 1+3 \alpha-6 \alpha^{2}+3 \alpha^{3}=1+\frac{3}{2}-\frac{3}{2}+\frac{3}{8}=\frac{11}{8}$. Therefore for $\alpha$ close to 0 or $\frac{1}{2}$ the first term in the expression approach infinity. Because $\frac{\epsilon^{\prime \prime}(a)}{\epsilon^{\prime}(a)}$ is assumed bounded the third term is bounded near both extremities, as is $\frac{d \log (V)}{d \alpha}$ since these converge smoothly to their limiting quantities as shown in the proof of Theorem 2 . Therefore the inequalities are always satisfied close to $\alpha=0$ and $\alpha=\frac{1}{2}$.

We conjecture that when there is a strong negative affiliation between $\sigma$ and $m$ and thus severe ironing (or even complete non-responsiveness) is necessary for small $\alpha$, non-concavities may arise as screening has no local benefits for small $a$ 's given non-responsiveness but may be globally optimal. However, we have yet to find an example where $W$ is not quasi-concave, despite considering a range of computational experiments where non-responsiveness is optimal for low $\alpha$. 


\section{References}

Armstrong, Mark, "Multiproduct Nonlinear Pricing," Econometrica, 64 (1996), 51-75.

Barder, Owen, Michael Kremer, and Ruth Levine, Making Markets for Vaccines: Ideas to Action. Washington, DC: Center for Global Development, 2005.

Chari, V. V., Mikhail Golosov, and Aleh Tsyvinski, "Prizes and Patents: Using Market Signals to Provide Incentives for Innovations," 2009.

http://www.dklevine.com/archive/refs4814577000000000398.pdf.

Chetty, Raj, "Sufficient Statistics for Welfare Analysis: A Bridge between Structural and Reduced-Form Methods;" Annual Review of Economics, 1 (2009), 451-488.

Cornelli, Francesca, and Mark Schankerman, "Patent Renewals and R\&D Incentives," Rand Journal of Economics, 30 (1999), 197-213.

Creedy, John, "The Principle of Transfers and The Variance of Logarithms," Oxford Bulletin of Economics 85 Statistics, 39 (1977), 153-158.

Dasgupta, Partha, Amartya Sen, and David Starrrett, "Notes on the Measurement of Inequality," Journal of Economic Theory, 6 (1973), 180-187.

"Leviathan Inc," The Economist, August 2010, 7th-13th.

Fabinger, Michael, and E. Glen Weyl, "A Multidimensional Envelope Theorem with Endogenous Choice Sets," in preparation, 2011.

Foster, James E., and Efe A. Ok, "Lorenz Dominance and the Variance of Logarithms," Econometrica, 67 (1999), 901-907.

Gallini, Nancy, and Suzanne Scotchmer, "Intellectual Property: When is it the Best Incentive System," Innovation Policy and the Economy, 2 (2002), 51-77.

Gilbert, Richard, and Carl Shapiro, "Optimal Patent Length and Breadth," Rand Journal of Economics, 21 (1990), 106-112.

Green, Jerry R., and Suzanne Scotchmer, "On the Division of Profit in Sequential Innovation," Rand Journal of Economics, 21 (1995), 20-33.

Guesnerie, Roger, and Jean-Jacques Laffont, "A Complete Solution to a Class of Principal Agent Problems with an Application to the Control of a Self-Managed Firm," Journal of Public Economics, 25 (1984), 329-629.

Hagood, John W., and Brian S. Thomson, "Recovering a Function from a Dini Derivative," American Mathematical Monthly, 113 (2006), 34-46.

Hopenhayn, Hugo, Gerard Llobet, and Matthew Mitchell, "Rewarding Sequential Innovations: Prizes, Patents, and Buyouts," Journal of Political Economy, 114 (2006), 1041-1068. 
Hopenhayn, Hugo A., and Matthew F. Mitchell, "Innovation Variety and Patent Breadth," Rand Journal of Economics, 32 (2001), 142-166.

Klemperer, Paul, "How Broad Should the Scope of Patent Protection Be?," Rand Journal of Economics, 21 (1990), 113-130.

Kremer, Michael, "Patent Buyouts: A Mechanism for Encouraging Innovation," Quarterly Journal of Economics, 113 (1998), 1137-1167.

Kremer, Michael, "Creating Markets for New Vaccines. Part i: Rationale," in Innovation Policy and the Economy, Adam B. Jaffe, Josh Lerner, and Scott Stern, eds., (Cambridge, MA: National Bureau of Economic Research, 200a), volume 1, 35-72.

Kremer, Michael, "Creating Markets for New Vaccines. Part ii: Design issues," in Innovation Policy and the Economy, Adam B. Jaffe, Josh Lerner, and Scott Stern, eds., (Cambridge, MA: National Bureau of Economic Research, 200b), volume 1, 73-118.

Marshall, Alfred, Principles of Political Economy, (New York: Macmillan, 1890).

McAfee, R. Preston, and John McMillan, "Multidimensional Incentive Compatibility and Mechanism Design," Journal of Economic Theory, 46 (1988), 335-354.

Milgrom, Paul R., and Ilya Segal, "Envelope Theorems for Arbitrary Choice Sets," Econometrica, 70 (2002), 583-601.

Milgrom, Paul R., and Chris Shannon, "Monotone Comparative Statics," Econometrica, 62 (1994), 157-180.

Milgrom, Paul R., and Robert J. Weber, "A Theory of Auctions and Competitive Bidding," Econometrica, 50 (1982), 1089-1122.

Mirrlees, James A., "An Exploration in the Theory of Optimum Income Taxation," Review of Economic Studies, 38 (1971), 175-208.

Mirrlees, James A., "The Implications of Moral Hazard for Optimal Insurance," mimeo, Nuffield College, (Conference in honour of Karl Borch, Bergen), 1979 .

O'Donoghue, Ted, Suzanne Scotchmer, and Jacques-François Thisse, "Patent Breadth, Patent Life, and the Pace of Technological Progress," Journal of Economics and Management Strategy, 7 (1998), 1-32.

Pigou, Arthur C., The Economics of Welfare, (London: MacMillan, 1920).

Piketty, Thomas, and Emmanuel Saez, "Income Inequality in the United States, 19131998," Quarterly Journal of Economics, 118 (2003), 1-39.

Rochet, Jean-Charles, and Philippe Choné, "Ironing, Sweeping and Multidimensional Screening," Econometrica, 66 (1998), 783-826.

Rogerson, William P., "The First-Order Approach to Principal-Agent Problems," Econometrica, 53 (1985), 1357-1367. 
Rosen, Sherwin, "Hedonic Prices and Implicit Markets: Product Differentiation in Pure Competition," Journal of Political Economy, 82 (1974), 34-55.

Royden, H. L., Real Analysis, Third Edition, (Englewood Cliffs, NJ: Prentice-Hall, 1988).

Scotchmer, Suzanne, "On the Optimality of the Patent Renewal System," Rand Journal of Economics, 30 (1999), 181-196.

Sen, Amartya, On Economic Inequality, (Oxford: Oxford University Press, 1973).

Shavell, Steven, and Tanguy van Ypersele, "Rewards versus Intellectual Property Rights," Journal of Law and Economics, 64 (2001), 525-547.

Smith, Adam, Lectures on Jurisprudence, (University of Glasgow, 1762).

Spence, Michael, "Job Market Signaling," Quarterly Journal of Economics, 87 (1973), $355-374$.

Toikka, Juuso, "Ironing Without Control," Journal of Economic Theory, 146, (2011), $2510-2526$.

Veiga, André, and E. Glen Weyl, "Multidimensional Product Design," 2012. http://www.glenweyl.com.

Weingarten, William F., "Empirical Calibration of Optimal Innovation Policy in Smartphone Application Markets," 2011, http://papers.ssrn.com/sol3/papers.cfm?abstract id $=1855823$.

Weyl, E. Glen, and Michal Fabinger, "A Restatement of the Theory of Monopoly," 2011. http://www.glenweyl.com.

E. Glen Weyl, and Jean Tirole, "Market Power Screens Willingness-to-Pay," 2011. http://idei.fr/doc/by/tirole/.

Wright, Brian D., "The Economics of Invention Incentives: Patents, Prizes, and Research Contracts," American Economic Review, 73 (1983), 691-707.

Young, W. H., and Grace Chisolm Young, "On the Discontinuities of Monotone Functions of Several Variables," Proceedings of the London Mathematical Society, 1924, s2-22 $(124-142)$. 